\title{
Article \\ Performance Characteristics of the Ultrasound Strategy during Incidence Screening in the UK Collaborative Trial of Ovarian Cancer Screening (UKCTOCS)
}

\author{
Jatinderpal Kalsi ${ }^{1}$, Aleksandra Gentry-Maharaj ${ }^{2}{ }^{-1}$, Andy Ryan ${ }^{2}$, Naveena Singh ${ }^{3}$, Matthew Burnell ${ }^{2}$, \\ Susan Massingham ${ }^{2}$, Sophia Apostolidou ${ }^{2}$, Aarti Sharma ${ }^{4}$, Karin Williamson $^{5}$, Mourad Seif ${ }^{6}$, Tim Mould $^{7}$, \\ Robert Woolas $^{8}$, Stephen Dobbs ${ }^{9}$, Simon Leeson ${ }^{10}{ }^{(0}$, Lesley Fallowfield ${ }^{11}$, Steven J. Skates ${ }^{12}$, Mahesh Parmar ${ }^{2}$, \\ Stuart Campbell ${ }^{13}$, Ian Jacobs ${ }^{1,14}$, Alistair McGuire ${ }^{15}$ and Usha Menon ${ }^{2, *}$ (i)
}

check for updates

Citation: Kalsi, J.; Gentry-Maharaj, A.; Ryan, A.; Singh, N.; Burnell, M.; Massingham, S.; Apostolidou, S.; Sharma, A.; Williamson, K.; Seif, M.; et al. Performance Characteristics of the Ultrasound Strategy during Incidence Screening in the UK Collaborative Trial of Ovarian Cancer Screening (UKCTOCS). Cancers 2021, 13, 858. https://doi.org/10.3390/ cancers 13040858

Academic Editor:

Sara Gutierrez-Enriquez

Received: 5 January 2021

Accepted: 9 February 2021

Published: 18 February 2021

Publisher's Note: MDPI stays neutral with regard to jurisdictional claims in published maps and institutional affiliations.

Copyright: (c) 2021 by the authors. Licensee MDPI, Basel, Switzerland. This article is an open access article distributed under the terms and conditions of the Creative Commons Attribution (CC BY) license (https:// creativecommons.org/licenses/by/ $4.0 /)$.
1 Department of Women's Cancer, Institute for Women's Health, University College London, London WC1E 6HU, UK; j.k.kalsi@ucl.ac.uk (J.K.); i.jacobs@unsw.edu.au (I.J.)

2 MRC Clinical Trials Unit at UCL, Institute of Clinical Trials \& Methodology, London WC1V 6LJ, UK; a.gentry-maharaj@ucl.ac.uk (A.G.-M.); a.ryan@ucl.ac.uk (A.R.); m.burnell@ucl.ac.uk (M.B.); s.massingham@ucl.ac.uk (S.M.); s.apostolidou@ucl.ac.uk (S.A.); m.parmar@ucl.ac.uk (M.P.)

3 Department of Pathology, Barts and the London, London E1 2ES, UK; Naveena.Singh@bartshealth.nhs.uk

4 Department of Obstetrics and Gynaecology, University Hospital of Wales, Cardiff CF14 4XW, UK; Aarti.Sharma@wales.nhs.uk

5 Department of Gynaecological Oncology, Nottingham City Hospital, Nottingham NG5 1PB, UK; Karin.Williamson@nuh.nhs.uk

6 Division of Gynaecology and of Cancer Services, St. Mary's Hospital and University of Manchester, Manchester M13 9WL, UK; Mourad.Seif@mft.nhs.uk

7 Department of Gynaecological Oncology, University College Hospital, London NW1 2BU, UK; tim.mould@uclh.nhs.uk

8 Department of Gynaecological Oncology, Queen Alexandra Hospital, Portsmouth PO6 3LY, UK; robert.woolas2@porthosp.nhs.uk

9 Department of Gynaecological Oncology, Belfast City Hospital, Belfast BT9 7AB, UK; Stephen.Dobbs@belfasttrust.hscni.net

10 Department of Obstetrics and Gynaecology, Ysbyty Gwynedd, Bangor, Gwynedd LL57 2PW, UK; simon.leeson@wales.nhs.uk

11 Cancer Research UK Sussex Psychosocial Oncology Group at Brighton \& Sussex Medical School, University of Sussex, Falmer BN1 9PX, UK; L.J.Fallowfield@sussex.ac.uk

12 Massachusetts General Hospital, Harvard Medical School, Boston, MA 02115, USA; sskates@gmail.com

13 Create Fertility Clinic, London EC2V 6ET, UK; profscampbell@hotmail.com

14 Department of Women's Health, University of New South Wales, Australia, Sydney 2052, Australia

15 London School of Economics and Political Science, London WC2A 2AE, UK; A.J.Mcguire@lse.ac.uk

* Correspondence: u.menon@ucl.ac.uk; Tel.: +44-7670-4909

Simple Summary: The United Kingdom Collaborative Trial of Ovarian Cancer Screening was undertaken to assess whether screening postmenopausal women from the general population might result in detection of ovarian/tubal cancers at an earlier stage and thus save lives. One of the screening strategies tested was a yearly transvaginal ultrasound scan of the ovaries (USS). Following the initial screen, 44,799 of the 50,639 women in the USS group went on to have a further 280,534 annual scans during April 2002-December 2011. Abnormalities leading to surgery were detected in 960 women of whom 113 (80 invasive epithelial) had ovarian/tubal cancer. Ovarian/tubal cancer was missed in 52 (50 invasive epithelial) women. Of the screen-detected cancers, $37.5 \%$ and missed cancers $6 \%$ were early stage(I/II). The number (detection rate $61.5 \% ; 80 / 130$ ) and advanced stage of the missed invasive cancers suggests that a yearly ultrasound scan may not be suitable for screening average risk women for ovarian cancer.

Abstract: Randomised controlled trials of ovarian cancer (OC) screening have not yet demonstrated an impact on disease mortality. Meanwhile, the screening data from clinical trials represents a rich resource to understand the performance of modalities used. We report here on incidence screening in the ultrasound arm of UKCTOCS. 44,799 of the 50,639 women who were randomised to annual screening with transvaginal ultrasound attended annual incidence screening between 28 April 2002 
and 31 December 2011. Transvaginal ultrasound was used both as the first and the second line test. Participants were followed up through electronic health record linkage and postal questionnaires. Out of 280,534 annual incidence screens, 960 women underwent screen-positive surgery. 113 had ovarian/tubal cancer (80 invasive epithelial). Of the screen-detected invasive epithelial cancers, 37.5\% (95\% CI: 26.9-49.0) were Stage I/II. An additional 52 (50 invasive epithelial) were diagnosed within one year of their last screen. Of the 50 interval epithelial cancers, $6.0 \%$ (95\% CI: 1.3-16.5) were Stage I/II. For detection of all ovarian/tubal cancers diagnosed within one year of screen, the sensitivity, specificity, and positive predictive values were $68.5 \%$ (95\% CI: 60.8-75.5), 99.7\% (95\% CI: 99.7-99.7), and $11.8 \%$ (95\% CI: 9.8-14) respectively. When the analysis was restricted to invasive epithelial cancers, sensitivity, specificity and positive predictive values were $61.5 \%$ (95\% CI: 52.6-69.9); 99.7\% (95\% CI: 99.7-99.7) and 8.3\% (95\% CI: 6.7-10.3), with 12 surgeries per screen positive. The low sensitivity coupled with the advanced stage of interval cancers suggests that ultrasound scanning as the first line test might not be suitable for population screening for ovarian cancer. Trial registration: ISRCTN22488978. Registered on 6 April 2000.

Keywords: ovarian cancer; screening; ultrasound; TVS; early detection; trial; randomised controlled trial; UKCTOCS

\section{Introduction}

Transvaginal ultrasonography (TVS) is considered the best modality for pelvic imaging, and is used routinely in the clinic for investigating women with suspected ovarian cancer. Based on its ability to assess ovarian volume and morphology, it has been used in large randomised trials of ovarian cancer screening as the primary screen. In the ovarian arm of the Prostate Lung Colorectal and Ovarian (PLCO) Cancer Screening trial [1], it was used in combination with the serum biomarker CA125 while in the ultrasound arm (USS) of the United Kingdom Collaborative Trial of Ovarian Cancer Screening (UKCTOCS), it was used as the sole primary screening test [2]. In both trials, there was no difference in the proportion of women detected with Stage I/II disease or deaths due to ovarian/tubal/peritoneal cancer between the ultrasound arm and the no screening (control) arm [2].

The data collected during these trials provides a rich resource to understand the performance characteristics of TVS in the setting of multicentre, general population screening. We have previously reported on the results of the initial (prevalence) USS screen [3]. We now report on the performance characteristics of USS screening in UKCTOCS during the 10 years of incidence screening.

\section{Results}

Following the initial (prevalence) screen, of the 50,639 women randomised to the USS arm 49,610 were eligible for incidence screening. Of them, 1029 were ineligible as both ovaries had been removed (896), death (131), moved away (2). Overall, 44,799 (88.5\%) of those randomized to the USS arm underwent incidence screening (Figure 1).

In total the women underwent 280,534 annual incidence screens between 28 April 2002 and 31 December 2011. Of these screens, 257,337 (91.8\%) were TVS, 20,707 (7.4\%) transabdominal, $2309(0.8 \%)$ both and for nine data on mode were missing. Individual women attended between 1 and 10 incidence screens with the median number per woman being 7 (IQR 5-8). The baseline characteristics of these women have been previously reported [2,3]. Median age of the women at the last annual incidence screen was 67 (IQR 62.6-72.0) years. 


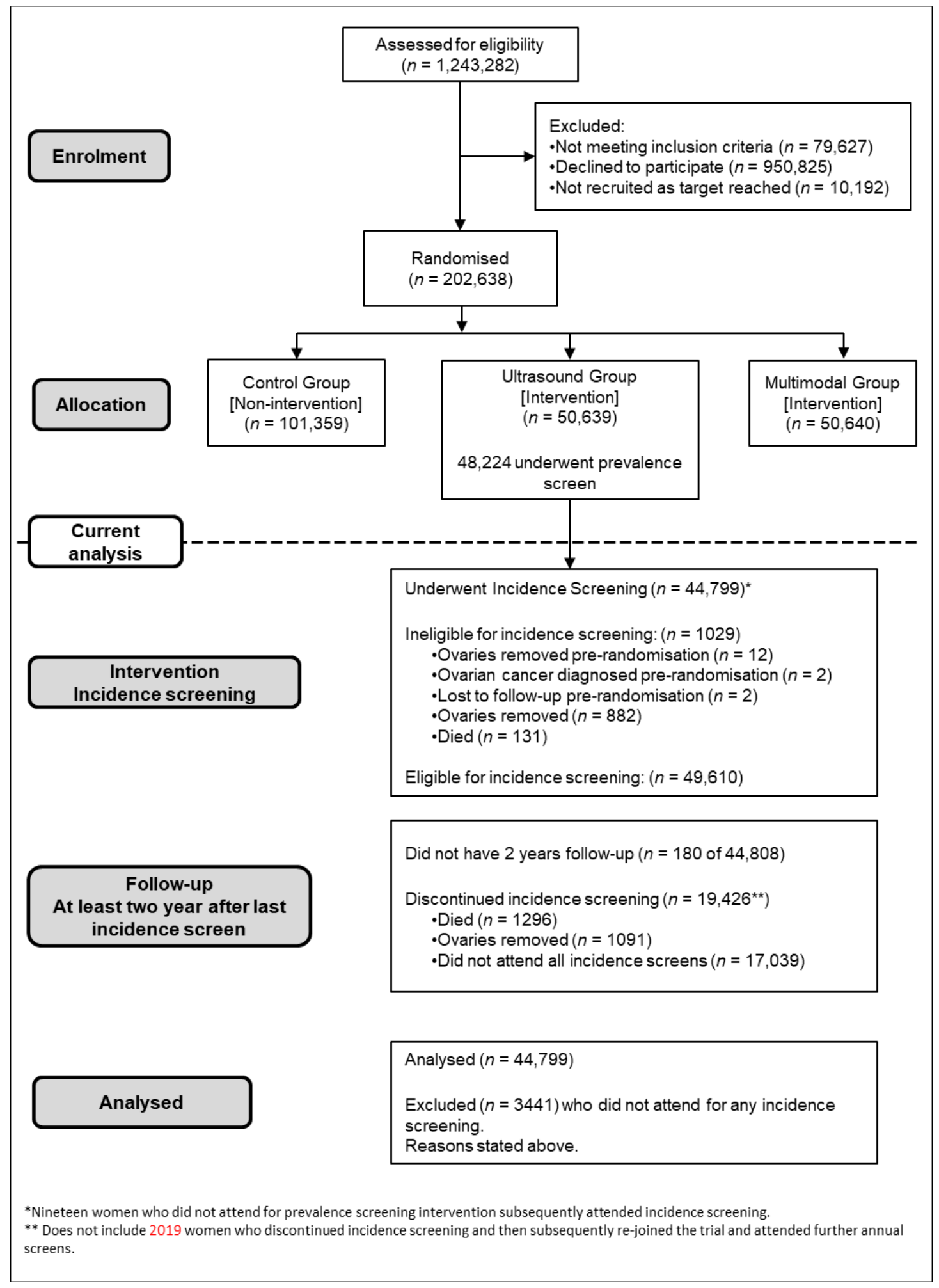

Figure 1. CONSORT diagram.

Overall, $99.4 \%(278,851 / 280,534)$ of the screens resulted in women being returned to annual screening. Two percent $(5497 / 280,534)$ of screens involving $4256(9.5 \% ; 4256 / 44,799)$ women resulted in referral for clinical evaluation. Of these women 960 ( $0.34 \%$ of screens; $960 / 280,534)$ were screen positive and had surgery (Figure 2 and Table 1). This figure includes one woman with a simple ovarian cyst who underwent surgery against protocol recommendation. 


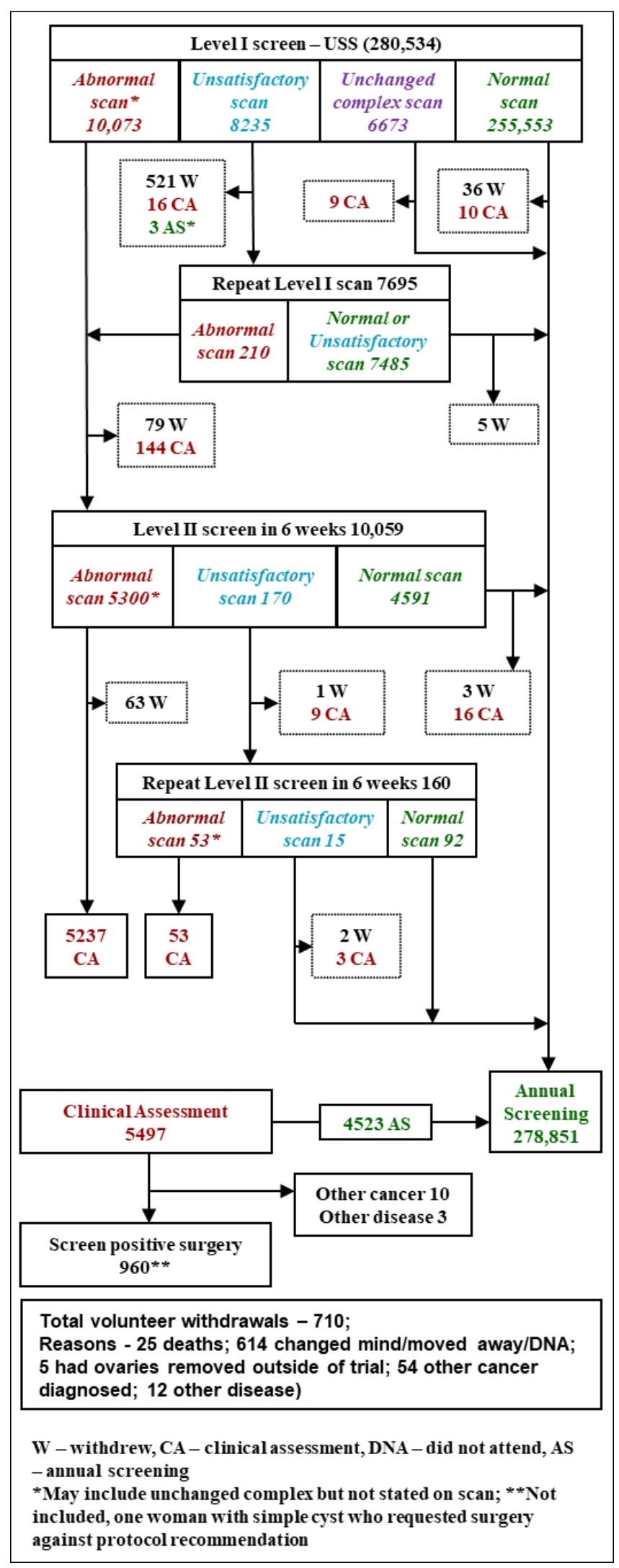

Figure 2. Ultrasound screening (USS) algorithm and outcome of incidence screening. 
Table 1. Results of annual incidence screens performed in USS group.

\begin{tabular}{|c|c|}
\hline Annual Incidence Screens & Women Years \\
\hline No. of Level 1 Screens * & $280,534(100)$ \\
\hline Normal Scan & $262,227(93.5)$ \\
\hline Unsatisfactory Scan & $8235(2.9)$ \\
\hline Abnormal Scan & $10,072(3.6)$ \\
\hline No. who Underwent Repeat Level 1 Screen $†$ & $7695(2.7)$ \\
\hline Returned to annual screening & $7485(97.3)$ \\
\hline Referred for Level 2 screen & $210(2.7)$ \\
\hline No. Who Underwent Level 2 Screen + & $10,060(3.6)$ \\
\hline Returned to annual screening & $4591(45.6)$ \\
\hline Referred for clinical assessment & $5299(52.7)$ \\
\hline Referred for Repeat Level 2 screen & $170(1.7)$ \\
\hline No. Who Underwent Repeat Level 2 Screen $†$ & $160(0.1)$ \\
\hline Returned to annual screening & $92(57.5)$ \\
\hline Referred for clinical assessment & $68(42.5)$ \\
\hline No. Referred for Clinical Assessment $†, \ddagger$ & $5495(2.0)$ \\
\hline No. Who Underwent Screen Positive Surgery + & $960(0.3)$ \\
\hline \multicolumn{2}{|l|}{ Surgical Approach } \\
\hline Diagnostic laparoscopy $\S$ & $31(3.2)$ \\
\hline Operative laparoscopy & $628(65.4)$ \\
\hline Combined laparoscopy and laparotomy & $69(7.2)$ \\
\hline Laparotomy & $214(22.3)$ \\
\hline Vaginal hysterectomy with BSO & $3(0.3)$ \\
\hline Imaging guided cytology/biopsy & $14(1.5)$ \\
\hline Missing data & $1(0.1)$ \\
\hline
\end{tabular}

Of the 960 surgical procedures, 69\% (662/960) were laparoscopic or vaginal. $113(11.8 \%)$ women were diagnosed with ovarian/tubal cancers (Table 2). This included 80 (70.8\%) invasive epithelial ovarian or tubal (iEOC), 29 (25.7\%) borderline (low malignant potential) epithelial ovarian, and 4 (3.5\%) non-epithelial ovarian cancers.

Table 2. Pathologic findings in screen positive women and those with interval cancers (screen negative).

\begin{tabular}{lc}
\hline Outcome of Screen Positive Surgery & All Women \\
\hline Total * & 960 \\
\hline Normal or benign pathology & 831 \\
Laparoscopy, ovaries normal, not removed & 24 \\
Normal ovaries & 91 \\
Benign ovarian pathology & 716 \\
Non-ovarian/tubal malignant neoplasms & 13 \\
Other non-ovarian cancer involving the ovaries(secondary ovarian neoplasm) & $7^{* *}$ \\
Other non-ovarian cancer not involving the ovaries & 6 \\
\hline
\end{tabular}

Screen Positive Women Diagnosed with Malignant Neoplasm of Ovary (ICD-C56) and Fallopian Tube (ICD-C57.0)

\begin{tabular}{lc}
\hline Total & $\mathbf{1 1 3}$ \\
\hline Non-epithelial neoplasm of ovary (ICD-C56) & 4 \\
Borderline epithelial neoplasm of ovary (ICD-C56) & 29 \\
Invasive epithelial neoplasm of tubo-ovarian origin (ICD-C56/C57.0) & 80 \\
\hline
\end{tabular}


Table 2. Cont.

\begin{tabular}{lc}
\hline Women with Screen Negative (Interval) Malignant Neoplasm of Ovary (ICD-C56) and \\
Fallopian Tube (ICD-C57.0) Diagnosed within One Year of End of Screen \\
\hline Total & 52 \\
\hline Borderline epithelial neoplasm of ovary (ICD-C56) & 2 \\
Invasive epithelial neoplasm of tubo-ovarian origin (ICD-C56/C57.0) & 50 \\
\hline
\end{tabular}

Data are numbers. ${ }^{*}$ Includes one volunteer who withdrew consent for accessing medical records and two volunteers where the ovaries were not identified due to extensive adhesions arising from a previous hysterectomies. ** Cancers of colorectal (3) breast (1), stomach (1), lymphoma (1), carcinoid small bowel (1).

Of the 29 borderline epithelial ovarian cancers, 28 (96.5\%) were Stage I/II as were 3 of $4(75 \%)$ non-epithelial ovarian cancers. Of the screen detected iEOC, $37.5 \%(30 / 80)$ were Stage I/II (Table 3). Of the iEOC $80 \%(64 / 80)$ were Type II and $18.8 \%(15 / 80)$ were Type I. Majority $(86.7 \% ; 13 / 15)$ of Type I were Stage I/II. Of Type II, only $26.6 \%(17 / 64)$ were Stage I/II. The median time from Level 1 annual screen to surgery for screen detected iEOC was 12.6 weeks (IQR 8.7 to 20.5 ).

Table 3. Stage and type of invasive epithelial ovarian and tubal cancers as per WHO 2014 classification.

\begin{tabular}{|c|c|c|}
\hline Characteristics & Positive & Negative \\
\hline \multirow[t]{2}{*}{ Total } & 80 & 50 \\
\hline & FIGO 2014 Stage & \\
\hline I & 18 & 2 \\
\hline II & 12 & 1 \\
\hline III & 45 & 26 \\
\hline IIIa & 5 & 0 \\
\hline $\mathrm{IIIb}$ & 13 & 3 \\
\hline IIIC & 27 & 23 \\
\hline IV & 5 & 21 \\
\hline \multirow[t]{2}{*}{ Early (I/II) stage-\%(95\% CI) } & $37.5(26.9,49.0)$ & $6.0(1.3,16.6)$ \\
\hline & Morphology & \\
\hline Type I iEOC (total) & $15(18.8 \%)$ & $1(2.0 \%)$ \\
\hline Low grade serous & 3 & 0 \\
\hline Endometrioid (low grade) & 3 & 0 \\
\hline Clear cell & 6 & 0 \\
\hline Mucinous & 3 & 1 \\
\hline Type II iEOC (total) & $64(80.0 \%)$ & $42(84.0 \%)$ \\
\hline High grade serous & 53 & 36 \\
\hline High grade endometrioid & 4 & 0 \\
\hline Carcinoma & 4 & 6 \\
\hline Carcinosarcoma & 3 & 0 \\
\hline Unclassified * & $1(1.3 \%)$ & $7(14.0 \%)$ \\
\hline
\end{tabular}

Date are numbers unless otherwise stated. ${ }^{*}$ Morphology could not be determined as only peritoneal fluid cytology was undertaken.

Of the 960 women who had screen positive surgery, 831 had benign pathology or normal adnexa (Table 2). In this subgroup, 35 (4.2\%) women had a major complication (with significant sequelae) (Table S1).

Median follow up from the end of incidence screening to cancer registration update in 2015 (25 March 2015 England and Wales, 15 April 2015 Northern Ireland) was 3.9 (IQR 3.6-5.0) years. Only 5 of $44,799(0.01 \%)$ women had follow-up of less than 2 years after their last screen. An additional 52 women were diagnosed with ovarian/tubal cancer (screen negative/interval cancer) within 1 year of the last incidence screen scan (Table 2). This included 2 borderline and $50 \mathrm{iEOC}$. Of the latter, $6 \%(3 / 50)$ were diagnosed at Stage I/II (Table 3). 
The sensitivity, specificity, and positive predictive values (PPV) were $68.5 \%$ (95\% CI: 60.8-75.5), 99.7\% (95\% CI: 99.7-99.7), and 11.8\% (95\% CI: 9.814) respectively for all ovarian and tubal cancers with 8.5 operations per case detected during incidence screening. When the analysis was restricted to iEOC, sensitivity, specificity and positive predictive values were 61.5\% (95\% CI: 52.6-69.9); 99.7\% (95\% CI: 99.7-99.7); and 8.3\% (95\% CI: 6.7-10.3) with 12 surgeries per screen positive (Table 4$)$.

Table 4. Performance characteristics of incidence USS screening for detection of ovarian and tubal cancers (WHO 2014 classification) within one year of screen.

\begin{tabular}{lc}
\hline Characteristics & No/\% (95\% CI) \\
\hline Number of women screen years & 280,534 \\
Number of surgeries & 960 \\
\hline Ovarian and Tubal Malignancies & \\
\hline Screen positives & 113 \\
Screen negatives & 52 \\
Sensitivity & $68.5 \%(60.8,75.5)$ \\
Specificity & $99.7 \%(99.7,99.7)$ \\
Positive predictive value & $11.8 \%(9.8,14.0)$ \\
No. of operations per screen positive & 8.5 \\
\hline Invasive Epithelial Ovarian and Tubal Malignancies * & 80 \\
\hline Screen positives & 50 \\
Screen negatives & $61.5 \%(52.6,69.9)$ \\
Sensitivity & $99.7 \%(99.7,99.7)$ \\
Specificity & $8.3 \%(6.7,10.3)$ \\
Positive predictive value & 12.0 \\
No. of operations per screen positive & \\
\hline Data are numbers or $\%$ (95\% CI) * excludes non epithelial and borderline epithelial ovarian neoplasms).
\end{tabular}

Data are numbers or \% $(95 \% \mathrm{CI})^{*}$ excludes non epithelial and borderline epithelial ovarian neoplasms).

Combining incidence and prevalence screening [3] of UKCTOCS, the sensitivity, specificity and positive predictive values were 72.3\% (95\% CI: 65.9-78.0), 99.5\% (99.5-99.5), and $9.1 \%$ (95\% CI: 7.8-10.5) for all ovarian and tubal cancers with 11.0 operations per case detected. When the analysis was restricted to iEOC, sensitivity, specificity, and positive predictive values were $63.3 \%(55.4,70.6), 99.5 \%$ (95\% CI: 99.5-99.5), and 5.8\% (4.78-7) with 17.2 surgeries per screen positive.

\section{Discussion}

\subsection{Principal Findings}

The performance characteristics of ultrasound screening in the largest ovarian cancer screening trial suggests that USS may not be suitable as a first line test for population screening. While the PPV was significantly higher $(11.8 \%$ vs. $5.3 \% ; p<0.0001)$ with fewer operations (8.5 vs. 18.8; $p<0.0001$ ) required to detect an ovarian/tubal cancer during incidence screening compared to the prevalence [3], the sensitivity was lower $(68.5 \%$ versus $84.9 \% ; p=0.02)$. For invasive epithelial cancers, while over one-third (38\%) of the screen detected invasive cancers were early stage, the majority $(94.0 \%)$ of the interval cancers were advanced (Stage III/IV). The latter, coupled with the low sensitivity $(61.5 \%)$ resulted in no overall difference ( $24 \%$ USS versus $26 \%$ Control; $p=0.57$ ) in low volume (Stage I, II, IIIa) invasive epithelial disease between USS and control arm on the previously reported intention to treat analysis [1,2].

\subsection{Results in Context}

While TVS is integral to all ovarian cancer screening strategies to date, its use as the primary screening test, as described here, has only been assessed in one other study, the University of Kentucky Ovarian Cancer Screening Trial (UKOCST). The latter study involved a slightly higher risk population with just under one fourth having a family 
history of ovarian and over $40 \%$ of breast cancer. It is a single arm single-centre prospective study and involved 46,101 women who underwent a mean of 6.5 annual screens [4]. Overall sensitivity for detecting ovarian cancers (85.5\% vs. $72 \%$ ) was higher than in the USS arm of our trial. TVS has a significant subjective component that is likely to be the key contributor to the differences noted. UKOCST involves a single centre, with all scans performed by a small group of highly experienced ultrasonologists. UKCTOCS involved over 200 Level I ultrasonologists [5] (certified sonographers or doctors with experience in gynaecological scanning in the National Health Service) across 13 centres undertaking $~ 45,000$ scans every year. The latter is more akin to a general population screening programme which would require annual scans for millions of women.

The sensitivity in our USS arm was significantly higher than that sensitivity of TVS alone $(44.6 \% ; 33 / 74)$ noted during four rounds of screening in the PLCO trial [6]. In the latter trial, the overall sensitivity was higher as the annual screen involved CA125 in addition to a scan, with abnormalities in both tests triggering additional investigations (combined strategy).

In comparison to a CA125-based strategy, PPV of ultrasound screening is low. The number of operations per ovarian cancer decreased from 18.8 during prevalence screening in our USS arm to 8.5 during incidence screening. This latter is similar to the 7.4 operations per case reported in the Kentucky study [4]. It is not possible to calculate a comparable estimate in the PLCO trial as a combined strategy was used.

In our trial, 10,000 complex adnexal masses were detected during the annual incidence screen. Through a process of repeat scanning for persistence of lesion and evaluation of ultrasound features by Level 2 expert sonologists, we were able to restrict surgery to just below 1000 of these women. Both the Kentucky and International Ovarian Tumour Analysis (IOTA) groups have over the years developed increasingly sophisticated rules/scoring systems to improve risk stratification of these adnexal masses and encourage conservative management. In the most recent international IOTA5 study of women with adnexal masses, they were able to avoid surgery in one-third on the basis of low risk ultrasound features [7].

A key requirement to impact on the high ovarian cancer mortality is detection of invasive epithelial ovarian/tubal cancer at a sufficiently early stage. A similar proportion of screen detected ovarian cancers were invasive epithelial both in our analysis (71\%: 80/113) and in the Kentucky study (75.5\%; 71/94). However, only 37.5\% (95\% CI: 26.9, 49.0) of screen detected invasive epithelial cancers were early stage (I and II) in our trial compared to $51 \%(45 / 71 ; 95 \%$ CI: 51.1, 74.5) in the latest report of the Kentucky study [4]. In the latter, this together with increased sensitivity is likely responsible for the significantly higher 5-year disease-specific survival of women with ovarian (including interval) cancers in the screening group (79 $\pm 4 \%$ ) compared to unscreened women with clinically detected epithelial ovarian cancer treated at the same centre during the same time period ( $45 \pm 2 \%$ ).

In comparison to a CA125 based approach [3], an ultrasound-based strategy detects a larger proportion of borderline ovarian cancers. This was similar in the Kentucky study (15.5\%; 17/124, 95\% CI: 9.3,23.6) and during incidence screening in UKCTOCS (18.8\%; $29 / 165,95 \%$ CI: $13.1,25.6)$. In our prevalence screen, it was higher $(37.7 \%, 20 / 53,95 \%$ CI: 24.7, 52.1). The lower incidence with time is likely due to increasing conservative management of less complex asymptomatic adnexal masses.

\subsection{Clinical and Research Implications}

The performance characteristics suggest that ultrasound as a first line test is not suitable for population ovarian cancer screening. The subjective nature of TVS, the challenges in identifying normal postmenopausal ovaries [8] that diminish in size with age and the low disease prevalence ( 1 in 2500) means that detection of disease early requires significant expertise coupled with constant attention to detail. In the course of the trial, we developed an accreditation programme for scanning postmenopausal ovaries [5]. However, our performance characteristics suggest that we were not able to replicate in the Level 1 ultrasonographers, the expertise available at a specialist centre such as Kentucky. The IOTA 
group have shown in multicentre studies that the performance of ultrasound prediction models/rules can be maintained in sonographers with varying levels of experience [9]. However, this is in the context of evaluation of adnexal masses, which is equivalent to a Level 2 rather than Level 1 screen during population screening. First line TVS screening of the population is always going to be a challenge given the size of the workforce required. The ideal is a less subjective, automated, and more reproducible test. In cervical screening, this has translated to HPV DNA testing increasingly replacing the older resource intensive and skill dependent cytology in many population-screening programmes.

Incidental adnexal findings are on the rise given the widespread use of ultrasound. The unnecessary surgery rates seen in our and the other ultrasound screening trials are relevant to the clinical management of these asymptomatic masses. Our findings suggest that many with low-risk features can be managed conservatively [10].

\subsection{Strengths and Limitations}

The key strengths of our study are the scale of the trial, high compliance with screening, the multicentre setting and detailed screening protocols and automated management algorithms, implemented by a dedicated central team. Completeness of data on screen-negative cancers was ensured by flagging of the trial cohort through cancer, death, and hospital administrative registries as well as postal follow-up of all women. All potential ovarian cancer cases were reviewed by an independent, blinded outcomes review committee.

A key limitation relates to use of self-reported visualisation of postmenopausal ovaries as a quality assurance measure during the trial. A retrospective audit of random, grey scale TVS images showed only moderate agreement for visualisation of normal ovaries between experts and sonographers and between expert reviewers alone [8]. This was despite a robust accreditation programme established within the trial for visualisation of postmenopausal ovaries. This again highlights the subjectivity of ultrasound scanning, use of video recordings of the ultrasound examination would probably have been a betterquality assurance measure. During the 14 years of trial, there have been significant advances in our understanding of the origin and heterogeneity of ovarian cancer. Our scanning protocol focused on evaluation of the ovary. However, we now know that at least half of high-grade serous cancers arise in the fallopian tube [11] making tubal evaluation critical. The Kentucky group has recently described and assessed such a protocol in older normal women and reported a 77\% visualisation rate [12]. Furthermore, in the last decade, there has been significant improvement in the resolution of ultrasound machines and their ability to detect subtle changes as a result of advances in ultrasound transducer technology and electronics.

\section{Materials and Methods}

\subsection{Ethical Approval}

The trial (ISRCTN22488978, ClinicalTrials.gov NCT00058032) was approved by the UK North West Multicentre Research Ethics Committees (North West MREC 00/8/34) with site specific approval from the local regional ethics committees and the Caldicott guardians (data controllers) of the primary care trusts. All participants provided written consent.

\subsection{Subjects and Screening Strategy}

The trial design has been described previously $[2,3,13]$. Briefly, 202,638 postmenopausal women aged 50 to 74 , from the general population were recruited through 13 regional trial centres located in NHS Trusts in England, Wales and Northern Ireland, between April 2001 and October 2005. Overall, 1.6\% of women had a maternal history of ovarian cancer and $6.3 \%$ a maternal history of breast cancer [3]. Women at increased risk of familial ovarian cancer were excluded from the study. The participants were randomised 1:1:2 to annual screening (until 31 December 2011) with serum CA125 (MMS: 50, 640) or TVS (USS: 50, 639) or no screening (control C: 101, 359). The full trial protocol is accessible at http:/ /ukctocs.mrcctu.ucl.ac.uk/media/1066/ukctocs-protocol_v90_19feb2020.pdf (ac- 
cessed on 4 February 2021). In the USS arm, 48,230 women underwent an initial (prevalence) screen [3].

Scans were performed by trial sonographers, the majority of whom worked in the NHS providing gynaecological scanning. All trial sonographers underwent additional training for assessment of postmenopausal ovaries and from 2008, formal accreditation [5]. Annual (Level 1) scans were performed by Type 1 (certified sonographers, trained midwives, or doctors with experience in gynaecological scanning) or Type 2 (experienced gynaecologists/radiologists, or senior sonographers, usually superintendent grade with particular expertise in gynaecological scanning) ultrasonographers. Repeat scans on detection of an abnormality (Level 2 scans) were only undertaken by Type 2 sonographers. Most scans during 2002-2008 were done on a dedicated Kretz SA9900 ultrasound machine (Medison, Seoul, Korea) and from 2008-2011 on Acquvix (Medison, Seoul, Korea).

At the annual transvaginal scan (Level 1), ovarian morphology and dimensions were assessed, and ovarian volume calculated. Ovarian morphology was classified as normal, simple cyst (single, thin walled, anechoic cyst with no septa or papillary projections) or complex (ovary had any non-uniform ovarian echogenicity excluding single simple or inclusion cyst). The number and size of cysts, wall regularity, presence and thickness of septae, size of papillations, and echogenicity of the fluid contents were recorded. The cysts were initially classified using the Kentucky screening trial morphology index [14] and from 2003, the International Ovarian Tumour Analysis (IOTA) classification [15]. Where an ovary was not visualised, the sonographer documented 'good view' if 3-5 cms of iliac vessels with well-defined walls and a clear anechoic centre was seen or 'poor view' and stated the reason such as bowel, fibroids, pelvic varicosities, or other. Ascites was defined as a vertical pool of fluid measuring $>10 \mathrm{~mm}$ in the Pouch of Douglas.

Ultrasound scans were classified based on the morphology of the adnexa and visualisation of the surrounding tissue as follows: (a) normal-where both ovaries had normal morphology or simple cysts were $<60 \mathrm{~cm}^{3}$, or were not visualised but a good view of the iliac vessels was obtained; (b) unsatisfactory-where one or both ovaries were not visualised due to a poor view); (c) abnormal - where one or both ovaries had complex morphology or simple cysts were $>60 \mathrm{~cm}^{3}$, or ascites was present. Based on these results the women were returned to annual screening (normal scan), repeat Level 1 scan (unsatisfactory scan) or Level 2 scan (abnormal scan). In women where adnexal masses had been previously managed conservatively and remained unchanged in morphology or volume (complex unchanged) on repeat annual screens, there was the option for clinical review of results and return to annual screening without undergoing Level 2. Women with an abnormal Level 2 scan were referred for clinical assessment.

This was undertaken at the regional centre by a designated trial clinician and included clinical evaluation and investigations as appropriate. Latter included serum CA125, repeat transvaginal scans and Doppler studies, CT/MRI of the abdomen and pelvis, and occasionally assessment of other tumour markers. A decision was made either to offer surgery or manage conservatively, taking into account the views of the woman, any significant comorbidity, morphological features of the ultrasound-detected lesion, previous hysterectomy, or major pelvic surgery that could contribute to false-positive ultrasound findings. The surgery in most cases involved removal of both ovaries and fallopian tubes using a laparoscopic approach where possible. If pelvic adhesions increased the risk of complications, the clinician could opt to remove only the 'abnormal' ovary. Hysterectomy was only undertaken where there was clear clinical indication. Women found to have ovarian or tubal cancer at a primary laparoscopic procedure underwent a subsequent staging procedure. Where there was high suspicion of ovarian cancer, laparotomy was undertaken. For those managed conservatively, the follow up plan usually involved a TVS and serum CA125 at 3 months with a possible repeat at 6 months, and return to annual screening if the findings were unchanged (unchanged complex). 


\subsection{Follow-Up}

Follow up involved electronic health record linkage for cancer and death registration and hospital admissions using the NHS number through the appropriate national agencies. Cancer registrations received until 25 March 2015 (England and Wales) and 15 April 2015 (Northern Ireland) were used for this analysis. In addition, women were sent postal questionnaires, 3-5 years post randomisation, and again in April 2014 after the end of screening [2].

\subsection{Confirmation of Diagnosis}

Copies of medical notes were retrieved for all women who had surgery as a consequence of a positive screening test as previously described [2]. Where cancer was diagnosed, additional information e.g., multidisciplinary team meeting notes, discharge summaries, and other relevant correspondence was also collated. The above were also obtained for all women where a notification was received either through linked electronic health records, follow-up questionnaire, or personal communication of a possible ovarian/tubal/peritoneal cancer. The case notes of all of these individuals were reviewed by an Outcomes Review Committee blinded to the randomisation group. They confirmed primary site, stage, morphology, and — where possible—classified invasive epithelial cancer into Type I (low-grade serous, low-grade endometrioid, mucinous, and clear cell cancers) or Type II (high-grade serous, high-grade endometrioid, carcinosarcomas and undifferentiated carcinoma) cancers [16]. Primary site was originally classified according to WHO 2003 [17] and more recently revised using WHO 2014 classification [18]. As a result, cancers initially classified as peritoneal have been reclassified for this analysis as ovarian/tubal. Stage for all cases included in this analysis have been re-reviewed by the Outcomes Committee and assigned as per FIGO 2014 criteria [19].

\subsection{Analysis}

This analysis is limited to annual screens that followed the initial (prevalence screen). An annual screen as previously defined is a single or series of scans culminating in surgery (screen positive) or return to annual screening (screen negative). For this analysis, women were censored at one year following the last scan performed as part of their last screening episode on the trial. The primary outcome measure was ovarian or fallopian tube cancer as per WHO 2014 classification [18] diagnosed within 12 months of the last scan. Sensitivity (proportion of ovarian/tubal cancers diagnosed within one year that were detected by screening), specificity (proportion of those without ovarian/tubal cancer who had a negative screen) and positive predictive value (proportion with a positive test result who actually had ovarian/tubal cancer) of incidence screening was calculated. Subgroup analysis of invasive epithelial cancers (borderline epithelial and non-epithelial ovarian cancers were excluded) was undertaken. Proportion of cancers detected in early (I/II) stage were calculated.

\section{Conclusions}

The performance characteristics suggest that ultrasound as the first line test may not be suitable for population screening.

Supplementary Materials: The following table is available online at https:/ /www.mdpi.com/2072 -6694/13/4/858/s1, Table S1: Surgical complications in women with benign adnexal masses.

Author Contributions: Conceptualisation-I.J., U.M., S.C., L.F., S.J.S., M.P., and A.M.; Data curationA.R. and U.M., Formal analysis-A.R., U.M., J.K., A.G.-M., and M.B.; Funding acquisition-I.J., U.M., M.P., L.F., S.J.S., S.C., and A.M.; Investigation-U.M., A.G.-M., A.R., A.S., K.W., M.S., T.M., R.W., S.D., S.L.; J.K., N.S., S.M., and S.A., Methodology-U.M., I.J., S.C., A.G.-M., A.R., and N.S., Project administration-U.M., J.K., A.G.-M., A.R., K.W., M.S., T.M., R.W., S.D., and S.L.; Resources-U.M., I.J., K.W., M.S., T.M., R.W., S.D., and S.L.; Software-U.M. and A.R.; Supervision-U.M., S.C., and I.J.; Validation-U.M., A.G.-M., A.R., S.M., A.S., and S.C.; Visualisation-A.R.; Writing—original draft 
J.K., A.G.-M., A.R., and U.M.; Writing—review and editing-all. All authors have read and agreed to the published version of the manuscript.

Funding: The current analysis is supported by National Institute for Health Research (NIHR) HTA grant (16/46/01) and The Eve Appeal. UKCTOCS was funded by Medical Research Council (G9901012 and G0801228), Cancer Research UK (C1479/A2884), and the Department of Health, with additional support from The Eve Appeal. Researchers at UCL are supported by the NIHR University College London Hospitals (UCLH) Biomedical Research Centre and MRC CTU at UCL core funding (MR_UU_12023).

Institutional Review Board Statement: The trial was conducted according to the guidelines of the Declaration of Helsinki, and Good Clinical Practice. The trial was approved by the UK North West Multicentre Research Ethics Committees (North West MREC 00/8/34) on 21 June 2000 with site-specific approval from the local regional ethics committees and the Caldicott guardians (data controllers) of the primary care trusts. The current trial protocol is located at http:/ / ukctocs.mrcctu. ucl.ac.uk/ukctocs / documents / (accessed date 1 Feberuray 2021).

Informed Consent Statement: All trial participants provided written informed consent.

Data Availability Statement: The datasets used and/or analysed during the current study are available from the corresponding author on reasonable request.

Acknowledgments: The authors thank the volunteers without whom the trial would not have been possible and everyone involved in conduct and oversight, especially the Ultrasound Sub-Committee (http:/ / ukctocs.mrcctu.ucl.ac.uk/ukctocs/committees/ (accessed date 1 Feberuray 2021)).

Conflicts of Interest: U.M. has stock ownership and has received research funding from Abcodia. I.J.J. reports personal fees from and stock ownership in Abcodia as the non-executive director and consultant. He has a patent for the Risk of Ovarian Cancer algorithm and an institutional license to Abcodia with royalty agreement. He is a trustee (2012-2014) and Emeritus Trustee (2015 to present) for The Eve Appeal. SJS reports personal fees from the LUNGevity Foundation and SISCAPA Assay Technologies as a member of their Scientific Advisory Boards, Abcodia as a consultant, and AstraZeneca as a speaker honorarium. He has a patent for the Risk of Ovarian Cancer algorithm and an institutional license to Abcodia. All other authors declare no competing interests.

Disclaimer: Views expressed in this publication are those of the authors and not necessarily those of the NHS, the NIHR or the Department of Health and Social Care.

\section{References}

1. Buys, S.S.; Partridge, E.; Black, A.; Johnson, C.C.; Lamerato, L.; Isaacs, C.; Reding, D.J.; Greenlee, R.T.; Yokochi, L.A.; Kessel, B.; et al. Effect of screening on ovarian cancer mortality: The Prostate, Lung, Colorectal and Ovarian (PLCO) Cancer Screening Randomized Controlled Trial. JAMA 2011, 305, 2295-2303. [CrossRef] [PubMed]

2. Jacobs, I.J.; Menon, U.; Ryan, A.; Gentry-Maharaj, A.; Burnell, M.; Kalsi, J.K.; Amso, N.N.; Apostolidou, S.; Benjamin, E.; Cruickshank, D.; et al. Ovarian cancer screening and mortality in the UK Collaborative Trial of Ovarian Cancer Screening (UKCTOCS): A randomised controlled trial. Lancet 2016, 387, 945-956. [CrossRef]

3. Menon, U.; Gentry-Maharaj, A.; Hallett, R.; Ryan, A.; Burnell, M.; Sharma, A.; Lewis, S.; Davies, S.; Philpott, S.; Lopes, A.; et al. Sensitivity and specificity of multimodal and ultrasound screening for ovarian cancer, and stage distribution of detected cancers: Results of the prevalence screen of the UK Collaborative Trial of Ovarian Cancer Screening (UKCTOCS). Lancet Oncol. 2009, 10, 327-340. [CrossRef]

4. $\quad$ van Nagell, J.R., Jr.; Burgess, B.T.; Miller, R.W.; Baldwin, L.; DeSimone, C.P.; Ueland, F.R.; Huang, B.; Chen, Q.; Kryscio, R.J.; Pavlik, E.J. Survival of Women With Type I and II Epithelial Ovarian Cancer Detected by Ultrasound Screening. Obstet. Gynecol. 2018, 132, 1091-1100. [CrossRef] [PubMed]

5. Sharma, A.; Burnell, M.; Gentry-Maharaj, A.; Campbell, S.; Amso, N.N.; Seif, M.W.; Fletcher, G.; Brunell, C.; Turner, G.; Rangar, R.; et al. Quality assurance and its impact on ovarian visualization rates in the multicenter United Kingdom Collaborative Trial of Ovarian Cancer Screening (UKCTOCS). Ultrasound Obstet. Gynecol. 2015, 47, 228-235. [CrossRef] [PubMed]

6. Partridge, E.E.; Kreimer, A.R.; Greenlee, R.T.; Williams, C.R.; Xu, J.-L.; Church, T.R.; Kessel, B.; Johnson, C.C.; Weissfeld, J.L.; Isaacs, C.; et al. Results From Four Rounds of Ovarian Cancer Screening in a Randomized Trial. Obstet. Gynecol. 2009, 113, 775-782. [CrossRef] [PubMed]

7. Froyman, W.; Landolfo, C.; De Cock, B.; Wynants, L.; Sladkevicius, P.; Testa, A.C.; Van Holsbeke, C.; Domali, E.; Fruscio, R.; Epstein, E.; et al. Risk of complications in patients with conservatively managed ovarian tumours (IOTA5): A 2-year interim analysis of a multicentre, prospective, cohort study. Lancet Oncol. 2019, 20, 448-458. [CrossRef] 
8. Stott, W.; Gentry-Maharaj, A.; Ryan, A.; Amso, N.; Seif, M.; Jones, C.; Jacobs, I.; Parmar, M.; Menon, U.; Campbell, S.; et al. Audit of transvaginal sonography of normal postmenopausal ovaries by sonographers from the United Kingdom Collaborative Trial of Ovarian Cancer Screening (UKCTOCS). F1000Research 2018, 7, 1241. [CrossRef] [PubMed]

9. Sayasneh, A.; Wynants, L.; Preisler, J.; Kaijser, J.; Johnson, S.; Stalder, C.; Husicka, R.; Abdallah, Y.; Raslan, F.; Drought, A.; et al. Multi-centre external validation of IOTA prediction models and RMI by operators with varied training. Br. J. Cancer 2013, 108, 2448-2454. [CrossRef] [PubMed]

10. Sharma, A.; Apostolidou, S.; Burnell, M.; Campbell, S.; Habib, M.; Gentry-Maharaj, A.; Amso, N.; Seif, M.W.; Fletcher, G.; Singh, N.; et al. Risk of epithelial ovarian cancer in asymptomatic women with ultrasound-detected ovarian masses: A prospective cohort study within the UK collaborative trial of ovarian cancer screening (UKCTOCS). Ultrasound Obstet. Gynecol. 2012, 40, 338-344. [CrossRef] [PubMed]

11. Soong, T.R.; Howitt, B.E.; Horowitz, N.; Nucci, M.R.; Crum, C.P. The fallopian tube, "precursor escape" and narrowing the knowledge gap to the origins of high-grade serous carcinoma. Gynecol. Oncol. 2019, 152, 426-433. [CrossRef] [PubMed]

12. Lefringhouse, J.R.; Neward, E.; Ueland, F.R.; Baldwin, L.A.; Miller, R.W.; DeSimone, C.P.; Kryscio, R.J.; van Nagell, J.R.; Pavlik, E.J. Probability of fallopian tube and ovarian detection with transvaginal ultrasonography in normal women. Womens Health 2016, 12, 303-311. [CrossRef] [PubMed]

13. Menon, U.; Gentry-Maharaj, A.; Ryan, A.; Sharma, A.; Burnell, M.; Hallett, R.; Lewis, S.; Lopez, A.; Godfrey, K.; Oram, D.; et al. Recruitment to multicentre trials-lessons from UKCTOCS: Descriptive study. BMJ 2008, 337, a2079. [CrossRef] [PubMed]

14. van Nagell, J.R., Jr.; DePriest, P.D.; Ueland, F.R.; DeSimone, C.P.; Cooper, A.L.; McDonald, J.M.; Pavlik, E.J.; Kryscio, R.J. Ovarian cancer screening with annual transvaginal sonography: Findings of 25,000 women screened. Cancer 2007, 109, 1887-1896. [CrossRef] [PubMed]

15. Timmerman, D.; Valentin, L.; Bourne, T.H.; Collins, W.P.; Verrelst, H.; Vergote, I. International Ovarian Tumor Analysis (IOTA) Group. Terms, definitions and measurements to describe the sonographic features of adnexal tumors: A consensus opinion from the International Ovarian Tumor Analysis (IOTA) Group. Ultrasound Obstet. Gynecol. 2000, 16, 500-505. [CrossRef] [PubMed]

16. Kurman, R.J.; Shih, I.-M. The Dualistic Model of Ovarian Carcinogenesis: Revisited, Revised, and Expanded. Am. J. Pathol. 2016, 186, 733-747. [CrossRef] [PubMed]

17. Tavassoli, F.A.; Devilee, P. (Eds.) Classification of Tumours, Pathology and Genetics: Tumors of the Breast and Female Genital Organs; World Health Organization: Lyon, France, 2003.

18. Daya, D.; Cheung, A.N.; Khunamornpong, S. Tumors of the peritoneum: Epithelial tumors of Müllerian type. In WHO Classification of Tumors of Female Reproductive Organs, 4th ed.; Kurman, R.J., Carcangiu, M.L., Herrington, C.S., Young, R.H., Eds.; International Agency for Research on Cancer: Lyon, France, 2014; pp. 92-93.

19. Prat, J.; FIGO Committee on Gynecologic Oncology. Staging classification for cancer of the ovary, fallopian tube, and peritoneum. Int. J. Gynecol. Obstet. 2014, 124, 1-5. [CrossRef] [PubMed] 